\title{
Acute exposure to stress improves performance in trace eyeblink conditioning and spatial learning tasks in healthy men
}

\author{
Roman Duncko, ${ }^{1,3}$ Brian Cornwell, ${ }^{2}$ Lihong Cui, ${ }^{1}$ Kathleen R. Merikangas, ${ }^{1}$ \\ Christian Grillon ${ }^{2}$ \\ ${ }^{1}$ Section on Developmental Genetic Epidemiology, Mood and Anxiety Disorders Program, National Institute of Mental Health, \\ $\mathrm{NIH}$, Bethesda, Maryland 20892, USA; ${ }^{2}$ Mood and Anxiety Disorders Program, National Institute of Mental Health, NIH, \\ Bethesda, Maryland 20892, USA
}

The present study investigated the effects of acute stress exposure on learning performance in humans using analogs of two paradigms frequently used in animals. Healthy male participants were exposed to the cold pressor test (CPT) procedure, i.e., insertion of the dominant hand into ice water for $60 \mathrm{sec}$. Following the CPT or the control procedure, participants completed a trace eyeblink conditioning task followed by a virtual navigation Morris water task (VNMWT). Hypothalamic-pituitary-adrenocortical (HPA) axis and sympathetic autonomic system (SAS) activity were assessed by measuring salivary cortisol, heart rate, and skin conductance at selected timepoints. Results revealed positive effects of stress on performance in both tasks. The stress group showed significantly more conditioned blinks than the control group during acquisition of trace eyeblink conditioning. The stress group also performed significantly better in the VNMWT than the control group, with the former showing significantly fewer failures to locate the hidden platform in the allotted time and smaller heading errors than the latter. Regression analyses revealed positive relationships between HPA axis and SAS activity during stress and eyeblink conditioning performance. Our results directly extend findings from animal studies and suggest potential physiological mechanisms underlying stress and learning.

Effects of stress on learning and memory have been extensively investigated in both humans and experimental animals (McGaugh 2000; Wolf 2003; Joels et al. 2006). Results are often contradictory, which may in part be due to differences in stress exposure (acute versus repeated) (Wolf 2003) and different components of memory analyzed (consolidation or short-term memory versus retrieval or long-term memory) (Roozendaal 2002; Het et al. 2005). Acute stress seems to facilitate memory formation when the learning procedure itself is arousing or emotionally valenced, or when learning takes place in a stressful context (Shors 2001; Bemelmans et al. 2003; Akirav et al. 2004; Jelici et al. 2004; Putman et al. 2004; Zorawski et al. 2005; Abercrombie et al. 2006; Andreano and Cahill 2006; Jackson et al. 2006; Steidl et al. 2006), but usually impairs retrieval (de Quervain et al. 1998; Domes et al. 2004). Repeated, or chronic, stress exposure is usually associated with deleterious effects on both memory consolidation and retrieval (Luine et al. 1994; Newcomer et al. 1999; Park et al. 2001; Wright and Conrad 2005). Importantly, even after focusing on effects of acute stress on short-term memory, there are contradicting findings showing impairment or no effect (Kirschbaum et al. 1996; Lupien et al. 1997; Woodson et al. 2003; Takahashi 2005).

Some of these inconsistencies may also be related to differences in how learning is often studied in animals versus human participants. In animals, learning performance is frequently assessed with classical conditioning (Shors 2001) and spatial navigation tests (Diamond et al. 1996; Woodson et al. 2003; Yang et al. 2003; Akirav et al. 2004), whereas only a few studies have

\footnotetext{
${ }^{3}$ Corresponding author.
}

E-mail dunckor@mail.nih.gov; fax (301) 480-2915.

Article is online at http://www.learnmem.org/cgi/doi/10.1101//m.483807. applied learning paradigms based on object recognition (Baker and Kim 2002). Learning and memory studies in humans have typically involved the use of word lists, picture sets, or short stories (Kirschbaum et al. 1996; Lupien et al. 1997; Bemelmans et al. 2003; Jelici et al. 2004; Putman et al. 2004; Takahashi 2005; Abercrombie et al. 2006; Andreano and Cahill 2006), but only rarely have they applied paradigms that directly translate to those used in animal studies (Zorawski et al. 2005; Jackson et al. 2006). Accordingly, the primary aim of the present study was to evaluate the acute effect of stress exposure on learning performance in human analogs of two paradigms frequently used in animals, eyeblink conditioning, and spatial navigation.

Eyeblink conditioning, a form of classical or Pavlovian conditioning, is widely used in experimental animals and has become an important tool for investigating learning and memory in human subjects because of our understanding of the underlying neural substrate (Christian and Thompson 2003). In humans, eyeblink conditioning involves repeated presentation of a conditioned stimulus (CS) (e.g., auditory tone) paired with an eyeblink-eliciting unconditioned stimulus (US) (e.g., air puff to eye). Learning is defined by the frequency in which the CS elicits conditioned eyeblink responses (CRs). Trace eyeblink conditioning is a specific form of eyeblink conditioning in which there is a temporal gap (e.g., 400-700 msec) between termination of the CS and onset of the US. Trace eyeblink conditioning is not only dependent on brain regions involved in delay eyeblink conditioning (i.e., no temporal gap between CS termination and US onset), such as the cerebellum, but also requires an intact hippocampus (Beylin et al. 2001; Kirsch et al. 2003), which may mediate declarative memory processes of the CS-US contingency (Clark et al. 2001). Acute stress exposure can have fast and longlasting facilitating effects on trace eyeblink conditioning in rats 
(Shors 2001); however, no complementary studies have been performed with human subjects.

The Morris water maze is another behavioral instrument employed to evaluate the effect of stress on learning in animals (de Quervain et al. 1998; Yang et al. 2003; Akirav et al. 2004). The procedure was originally developed to assess the effects of hippocampal lesions on spatial navigation in rats (Morris et al. 1982). Briefly, a rat is placed in a circular pool of opaque water with the only escape being a platform that is submerged and not visible. Under these highly stressful conditions, learning involves encoding and maintenance of spatial cues in the distal environment to recall and navigate to the location of the platform from trial to trial. This task has been extensively applied as a highly sensitive and specific tool for investigating hippocampal involvement in spatial learning (D'Hooge and De Deyn 2001). Recently, a computer-generated virtual reality version of this test has been developed for studies in human subjects (Hamilton et al. 2002). Performance on the virtual navigation Morris water task (VNMWT) is sensitive to variables associated with spatial memory and reasoning abilities (e.g., age, gender) and has also been shown to be dependent on hippocampal processes (Driscoll et al. 2005). In rodents, exposure to acute stress affects short-term memory performance during spatial navigation (Diamond et al. 1996; Woodson et al. 2003; Akirav et al. 2004). It is presently unknown whether acute stress has effects on spatial navigation in humans.

In contrast to the original description of the stress response as generalized and uniform (Selye 1975), it is now viewed as more complex and variable, depending on the nature of the stressor and the current status of the stressed subject (Kopin 1995; Pacak and Palkovits 2001; Duncko et al. 2006). The main organ systems involved in these physiological responses are the sympathetic autonomic system (SAS) and the hypothalamic-pituitaryadrenocortical (HPA) axis. The SAS is involved in regulation of cardiovascular, metabolic, and thermoregulatory functions through release of catecholamines from the adrenal medulla and sympathetic nerve endings, whereas the HPA axis acts primarily through release of glucocorticoids from the adrenal cortex (Habib et al. 2001). Both groups of hormones affect learning and memory (McGaugh 2000; Wolf 2003). It has been suggested that a concerted action of glucocorticoids and catecholamines is required for a maximal effect on learning (Cahill et al. 2003; Roozendaal et al. 2004a; Joels et al. 2006).

Different types of stress stimuli may induce different activations of individual components of the stress response (e.g., SAS and HPA axis) and may result in different effects on learning and memory, potentially leading to inconsistent findings reported in the literature. Our secondary aim was to investigate the link between stress-related changes in learning performance and SAS and HPA activation. HPA axis activity was measured by analyzing cortisol concentrations in saliva, and SAS activity was assessed by heart rate (HR) and skin conductance measurements.

\section{Results}

\section{Trace eyeblink conditioning}

The CR rate significantly increased from block 1 to block 6 of acquisition $\left(F_{(1,26)}=8.4, P<0.01\right)$. The stress group emitted significantly more CRs during acquisition relative to the control group $\left(F_{(1,26)}=4.7, P<0.05\right)$ (Fig. 1$)$. No significant group differences were observed in the number of spontaneous eyeblinks during acquisition (control: $6.4 \pm 0.8 \%$, stress: $7.4 \pm 1.6 \%$, $F<1$ ) or in the amplitude of unconditioned responses during the first four trials of the habituation phase (control: $46.6 \pm 10.2 \mu \mathrm{V}$, stress: $50.1 \pm 5.2 \mu \mathrm{V}, F<1$ ). No significant group difference was

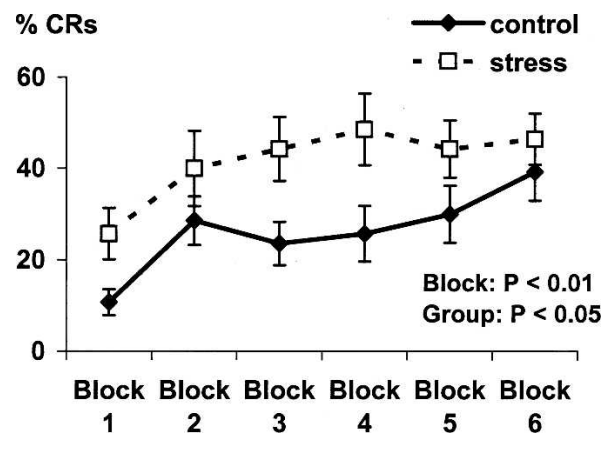

Figure 1. Percentage of possible CRs during six blocks of acquisition. Data are expressed as mean \pm SEM. $P$-value reflects the statistical significance of within and between group differences.

found in the number of CRs during extinction (control: $13.9 \pm 3.2 \%$, stress: $15 \pm 3.4 \%, F<1)$.

\section{Virtual navigation Morris water task performance}

The stress group performed significantly better in the VNMWT, with significantly fewer failures to find the platform $\left(t_{(26)}=2.3\right.$, $P<0.05)$ and significantly smaller heading errors $\left(F_{(1,26)}=5.0\right.$, $P<0.05)$, compared with the control group. Group differences in latency to reach the platform and path length showed a similar pattern but failed to reach statistical significance $\left(F_{(1,26)}=3.1\right.$, $P=\mathrm{NS}$ and $F_{(1,26)}=1.4, P=\mathrm{NS}$, respectively) (Fig. 2). Main effects of block were found for each performance variable (heading error: $F_{(1,26)}=4.1, P<0.01$; latency: $F_{(1,26)}=8.1, P<0.01$; path length: $\left.F_{(1,26)}=6.97, P<0.01\right)$, with no interactions between group and block.

\section{Stress response}

Exposure to the cold pressor procedure was associated with the subjective experience of moderate to severe pain (3.8 \pm 0.1 arbitrary units). Analysis of neuroendocrine changes (HR, skin conductance responses [SCRs], salivary cortisol) during the cold pressor stress procedure revealed significant time $\times$ group interactions at timepoint 0 min after stress exposure for $\mathrm{HR}$ $\left(F_{(1,25)}=14.3, P<0.01\right)$ and $\operatorname{SCR}\left(F_{(1,20)}=7.5, P<0.05\right)$. This indicates that the stress group had significantly higher HR and SCR during the period of cold water immersion than the control group (Fig. 3). No significant group difference was observed for salivary cortisol concentrations $(F<1)$.

\section{Associations between stress response and learning performance}

Regression analysis revealed that neither HR $(F=2.8, P=$ NS, $\left.r^{2}=0.19\right)$ nor SCR $\left(F=2.3, P=\mathrm{NS}, r^{2}=0.17\right)$ alone predicted the eyeblink conditioning performance. However, with inclusion of peak cortisol concentrations in the analysis, the model combining HR and cortisol significantly predicted the rate of CRs $(F=5.97, P<0.05)$, with both predictors having similar positive relationships with learning performance $\left(\beta_{\text {heart rate }}=0.49\right.$, $\beta_{\text {cortisol }}=0.53, r^{2}=0.54$ ) (Fig. 4). The model combining SCR and cortisol produced a similar outcome, but the overall predictive value did not reach statistical significance $\left(\beta_{\mathrm{SCR}}=0.32\right.$, $\beta_{\text {cortisol }}=0.45, F=2.58, P=\mathrm{NS}, r^{2}=0.36$ ). No such associations were observed with the number of spontaneous eyeblinks or unconditioned response amplitudes as dependent variables. Learning performance on the VNMWT defined by mean latency, mean path length, and mean heading error was not predicted by any of the stress response measures. 

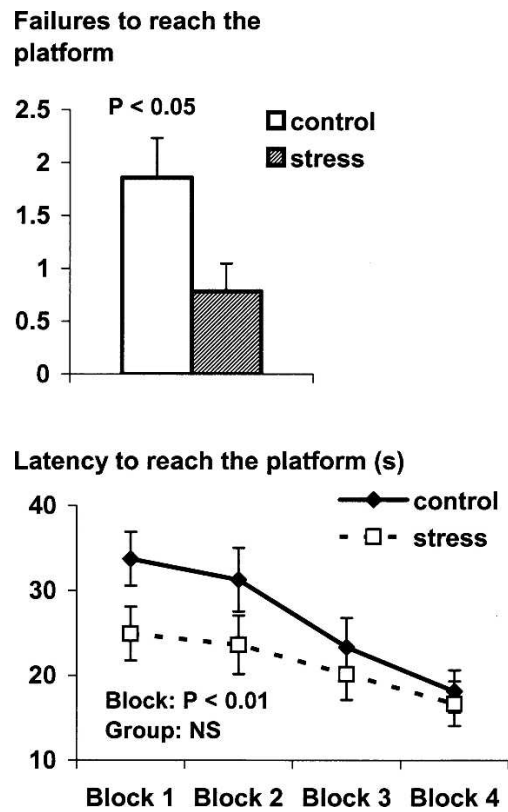
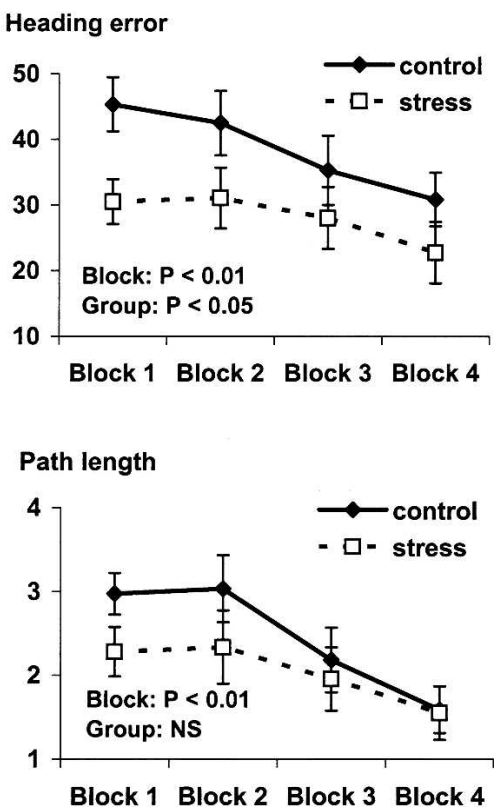

Figure 2. Learning performance during the virtual navigation Morris water task as described by number of failures, heading error, latency to reach the platform, and path length. Data are expressed as mean \pm SEM. $P$-values reflect the statistical significance of within and between group differences; NS indicates not significant.

\section{Discussion}

The present findings document that acute exposure to cold pressor stress was associated with improved performance in both learning paradigms. To our knowledge, this is the first report of effects of acute stress exposure on trace eyeblink conditioning and virtual spatial navigation in human participants. The higher CR acquisition rate after exposure to the cold pressor test directly extends findings of improved eyeblink conditioning after stress in animals (Shors 2001). The facilitating effect of stress on eyeblink conditioning does not appear to be attributable to nonassociative processes for two primary reasons. First, the stress and control group did not differ in UR magnitude, a variable sensitive to current autonomic arousal (Tracy et al. 2005) and known to affect the rate of CRs (Grillon and Hill 2003). Second, spontaneous eyeblink rates did not differ between groups, giving us assurance that the stress group did not show a nonspecific increase in blinking, which could have artificially inflated CR rates in this group. Instead, our results most likely reflect facilitation of associative processes mediating eyeblink conditioning as a function of prior exposure to stress.

This finding is consistent with animal data (Shors 2001) and with human studies showing improved performance in other forms of associative learning paradigms after exposure to acute stress (Zorawski et al. 2005; Jackson et al. 2006). Results from animal studies indicate that the stress-induced facilitation of eyeblink conditioning might be mediated by a circuit including basolateral amygdala (Shors and Mathew 1998), bed nucleus of the stria terminalis (Bangasser et al. 2005), and hippocampus (Shors 2001; Shors et al. 2001; Weiss et al. 2005). The neurotransmitters implicated include corticotropin-releasing hormone (CRH) (Servatius et al. 2005) and glutamate (Shors and Mathew 1998). It has been suggested that the $N$-methyl-D-aspartate (NMDA) receptor activation in basolateral amygdala may facilitate learning by enhancing the neural representation of relevant cues (Shors and Matzel 1997; Shors and Mathew 1998), but the role of such mechanisms in humans still remains to be elucidated.

The improved learning performance during the VNMWT is driven by superior performance of the stressed group during the first block of trials (see Fig. 2). Because participants were presented on the first trial with a visible platform to promote encoding, the effects of stress may have, in part, facilitated encoding and subsequent navigation to the hidden platform during the first block of test trials (D'Hooge and De Deyn 2001). General arousal and motor activation may also be contributing to this early performance advantage in the stressed group, although it is not clear how these nonspecific processes could specifically influence path direction and lead to smaller (i.e., better) heading errors in the stressed group. More careful manipulation of the parameters of the virtual water maze task will be necessary to elucidate which components underlying performance on this task may be affected by acute stress exposure.

Effects of stress on cognitive functions have been suggested to be mediated by several mechanisms (Croiset et al. 2000; McGaugh 2000; Servatius and Beck 2003). The present study focused on the HPA axis and the SAS. HPA axis activation is represented by concentrations of cortisol measured in saliva. Cortisol enters saliva by diffusion and has been shown to reliably reflect the concentrations of unbound cortisol in blood (Vining et al. 1983). Cortisol is the main product of HPA axis activation during stress, playing a major role in mediating effects of stress on cognitive functions, particularly through its action on receptors located in the hippocampus, amygdale, and prefrontal cortex (Herman et al. 1989; Morimoto et al. 1996; Roozendaal et al. 2004b). SAS activity was indirectly assessed by measuring HR and skin conductance. Although peripheral SAS activity is known to be associated with cognitive and behavioral changes during stress, the underlying mechanisms are not completely understood. One mechanism is probably related to centrally coordinated activity of brainstem nuclei involved in central release of norepinephrine (NE) and those involved in the regulation of SAS and peripheral release of NE. Another pathway seems to be related to peripheral $\mathrm{NE}$ acting on beta adrenergic receptors localized on vagal afferent fibers, resulting in vagal stimulation and central NE release (Hassert et al. 2004). Indeed, vagal activation has been shown to be 


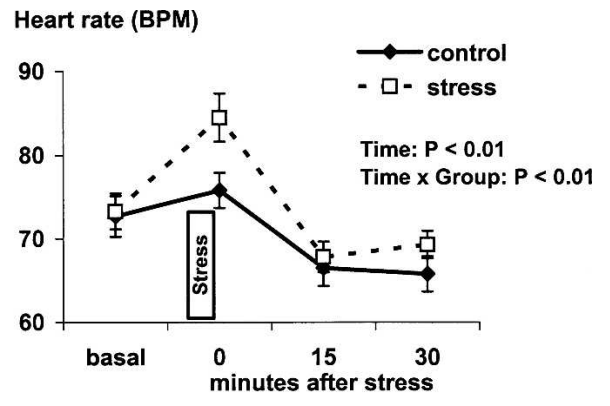

SCR (fluctuations/10s)
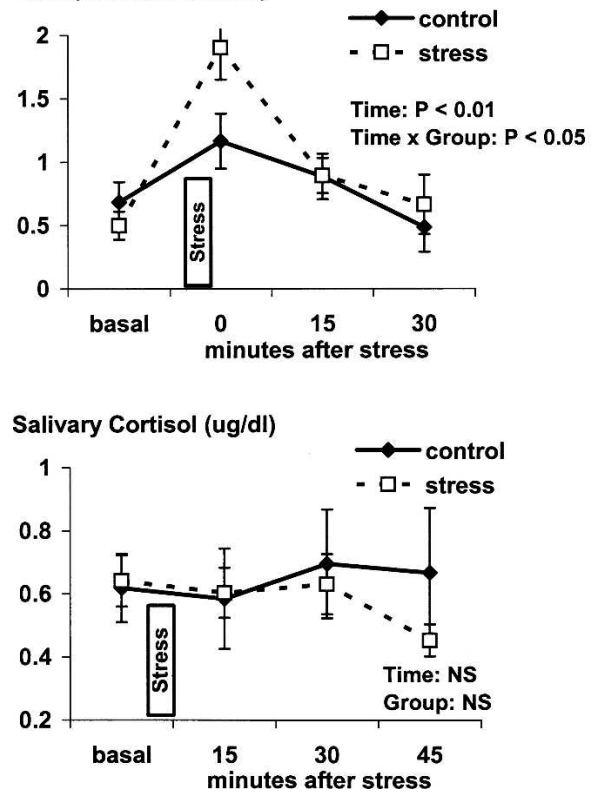

Figure 3. Heart rate, skin conductance responses (SCR), and salivary cortisol at timepoints before, during, and after the cold pressor stress procedure. Data are expressed as mean \pm SEM. $P$-value reflects the statistical significance of main effect of time and time $\times$ group interaction at the timepoint 0 min after stress; NS indicates not significant.

associated with improved eyeblink conditioning (Tapp et al. 1997).

The 1-min duration cold stress procedure applied in the present study was associated with perception of moderate to severe pain and marked variability in SAS activation, with HPA activation found in only a subset of subjects. This is consistent with other studies using a similar cold pressor procedure (van Eekelen et al. 2003; McRae et al. 2006), suggesting that this test is mildly aversive. Lengthening the duration of the cold pressor test may lead to more homogeneous and stronger HPA axis activation across individual subjects. Indeed, Cahill et al. (2003) found a marked increase in salivary cortisol after a 3-min cold pressor test. To capitalize on this variability, we performed linear regression analyses to identify potential relationships between the criterion variable of eyeblink CR rates and the predictor variables of HPA axis and SAS responses following stress exposure. Analyses revealed that high activity of both components of stress response was strongly associated with maximal learning performance. This observation reinforces the claim that both HPA axis activation and NE release are required for an optimal effect on learning (Roozendaal et al. 1996, 2006; Cahill et al. 2003; Okuda et al. 2004; Abercrombie et al. 2005) and supports the concept of convergence in time and space formulated by Joels et al. (2006).

The findings described in the present study should be inter- preted in the context of the strengths and limitations of this study. Major strengths are that the methods for assessing learning translate directly to animal tasks and that we made a preliminary attempt at linking learning performance following stress exposure to measures of HPA and SAS activity. Although we recognize that there are intrinsic differences between human and animal experimentation that cannot be controlled, the tasks used here share much of the same structure as those used with animals, leading us to the tenable assumption that a similar set of cognitive processes is being studied in both cases. One major limitation of the study involves the two tasks not being counterbalanced, which is why we remain cautious in comparing the effects of cold-pressor stress on spatial navigation in the virtual maze task versus eyeblink conditioning. Moreover, to the extent that the eyeblink conditioning procedure was aversive, virtual maze performance may have been jointly influenced by exposure to the air puffs as well as the cold pressor. However, it is unlikely that the eyeblink conditioning task was more aversive than the water maze task primarily because measures of SAS activation obtained during both learning tasks did not show consistent differences. Despite the lack of task counterbalancing, we find convergent results across these two different learning tasks to be a compelling demonstration of the positive effects of acute stress exposure on learning in healthy men. As most animal studies on stress and learning are performed on male animals, focusing on healthy men also facilitated comparison of our findings to those in the animal literature. Further, because the effect of stress on learning is known to be modulated by sex (Wolf 2003), exclusion of healthy women allowed us to reach sufficient power with fewer participants. Exploring the role of gender in modulating these effects in humans is critical to a more complete understanding of how stress affects learning.

In conclusion, our study extends findings from animal studies, showing that acute exposure to the cold pressor stress facilitates eyeblink conditioning as well as spatial navigation performance in healthy men. Both HPA axis and SAS activation during stress may be critical for maximal positive impact of acute stress exposure on eyeblink conditioning. Neuroendocrine mediation of the effect of stress on virtual navigation performance seems to be more complex. Further research applying neuroimaging methods as well as studies focusing on affected populations are

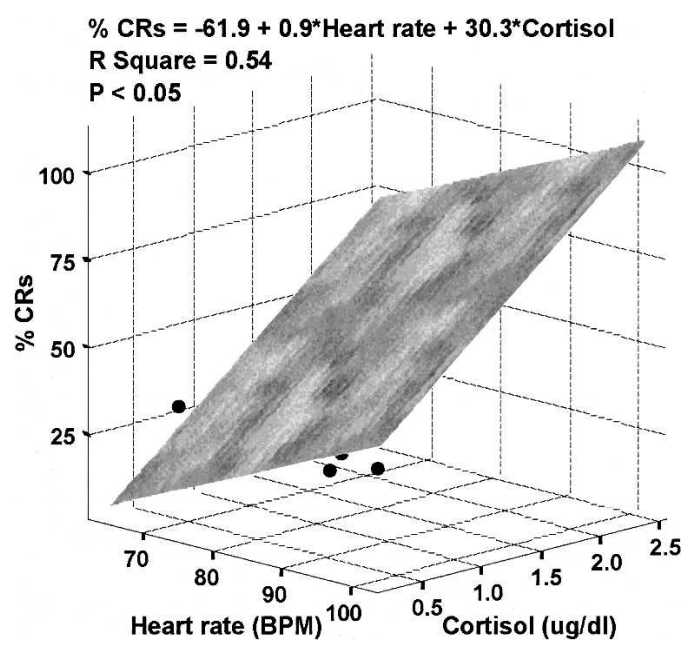

Figure 4. Three-dimensional scatterplot (with least-squares plane) showing the overall predictive relationship of heart rate during stress $(X$-axis) and peak salivary cortisol levels (Z-axis) with the rate of conditioned responses (\% CRs, $Y$-axis) during trace eyeblink conditioning. $P$ value reflects the statistical significance of the regression model.

\section{Learning \& Memory www.learnmem.org}


expected to improve our understanding of neural circuits and structures underlying stress and learning.

\section{Materials and Methods}

\section{Participants}

Thirty-three physically and mentally healthy volunteers were recruited through ads in local newspapers. To control for confounding effects of gender and age, only male participants younger than $40 \mathrm{yr}$ were included in this study. All participants underwent a screening procedure including a physical examination, the structured clinical interview for DSM-IV (First et al. 1995), and urine toxicology analysis to examine their health status. Only participants free of a medical condition, past or current psychiatric disorders, and current use of drugs or psychoactive medications were invited to participate in the study. Previous experiences with eyeblink conditioning and/or the virtual water maze test were also exclusion criteria. Data obtained from five participants had to be excluded due to technical problems during eyeblink conditioning. The final sample included 28 participants (age: $25.7 \pm 4.8 \mathrm{yr}$ ) randomly assigned to a control or stress group with 14 participants per group. All participants signed an informed consent form approved by the National Institute of Mental Health (NIMH) Human Investigation Review Board.

\section{Procedure}

All testing sessions were conducted in the morning between 0800 and $1100 \mathrm{~h}$. Following informed consent procedures, electrodes for SCR and HR recording were attached, followed by a 10-min basal recording of SCRs and HR.

Participants assigned to a stress group underwent the cold pressor test procedure lasting $6 \mathrm{~min}$, while the control group remained sitting in the experimental room. The cold pressor procedure consisted of immersion of the dominant hand for $5 \mathrm{~min}$ into room temperature water $\left(23^{\circ} \mathrm{C}\right)$, followed immediately by immersion for $1 \mathrm{~min}$ into ice water $\left(0-2^{\circ} \mathrm{C}\right)$ produced by mixing $2 \mathrm{~L}$ of tap water with the same amount of crushed ice. Air pump tubing was inserted into both room temperature water and ice water containers to ensure water circulation and constant temperature throughout the container. Within $5 \mathrm{~min}$ after the cold pressor test, participants were asked to report the subjective painfulness of the procedure by using a five-point Likert scale $(1$, no pain; 2 , mild pain; 3 , moderate pain; 4 , severe pain; 5 , overwhelming pain).

\section{Single-cue trace eyeblink conditioning}

Fifteen minutes after the cold water procedure, participants were prepared for the eyeblink conditioning. Two standard disk electrodes were attached to the skin near the right eye, one just above the infraorbital ridge parallel to the pupil and the other just lateral to the temporal cantus. Impedance was kept $<5 \mathrm{k} \Omega$. The US was an air puff (10 psi, $100 \mathrm{msec})$ delivered to the inner eye corner by air tubing attached to standard protective goggles. A pure tone $(1000 \mathrm{~Hz}, 75 \mathrm{~dB}, 400 \mathrm{msec})$ delivered binaurally through headphones was used as the CS.

The conditioning procedure consisted of habituation, acquisition, and extinction phases. During habituation, four isolated USs and five unreinforced CSs were presented. The acquisition phase consisted of six blocks containing 10 trials. The first nine trials in each block contained the CS paired with the US with a 600-msec trace interval (stimulus onset asynchrony: $1000 \mathrm{msec}$ ). For the 10th trial within each block, an unreinforced CS was presented. The extinction phase consisted of two blocks of 10 unreinforced CS presentations. Intertrial intervals throughout the procedure were 8-12 sec with an average of $10 \mathrm{sec}$.

For analysis of acquisition data, CRs were defined as eyeblink responses with onsets $\leq 400 \mathrm{msec}$ before the US presentation. As the long trace interval increases the risk of spontaneous blink to be counted as CRs, we assessed whether the rate of CRs was greater than the rate of spontaneous blinks and whether there was an effect of treatment (stressor) on spontaneous blinks. Spontaneous blinks were defined as those that occurred in the 500-msec interval before CS presentation. CRs and spontaneous blinks are presented as percentage of possible responses.

\section{Virtual navigation Morris water task}

Thirty minutes after the cold water procedure, participants underwent the VNMWT. During this task, a virtual environment consisting of a circular pool located in a square room with four distal visual cues on the walls was presented on a computer screen (Driscoll et al. 2005). Using arrow keys, participants were instructed to navigate through the pool and to find the platform as fast as possible even if it was not visible. After getting accustomed to the controls, participants were presented a trial with the platform visible, followed by 16 trials with the platform not visible. During those trials, the platform position remained constant but starting locations differed from trial to trial. If the participant failed to locate the platform within $60 \mathrm{sec}$, the platform became visible and the participant was prompted to navigate to it as quickly as possible.

For analysis of performance, latency to reach the platform, length of path traveled relative to the pool diameter, and heading error (the angle between the ideal and actual path trajectory) were averaged across four consecutive trials, creating four blocks. These performance variables were calculated automatically by the software. The total number of failures to reach the platform in the allotted $60 \mathrm{sec}$ was also calculated for each participant.

\section{Heart rate and skin conductance responses}

$\mathrm{HR}$ is presented in beats per minute and was calculated from interbeat intervals recorded during 5-min periods at the beginning of baseline, during eyeblink conditioning, and virtual water maze navigation. HR was also measured for 1 min during cold water immersion in the stress group and for 1 min during the corresponding interval in the control group.

SCRs, defined as spontaneous increase in skin conductance exceeding 0.05 microSiemens $(\mu \mathrm{S})$, were analyzed during the same periods as HR and are presented as number of responses per 10 sec.

\section{Saliva collection}

Saliva samples were obtained with the use of plain cotton swab Salivettes. Samples were taken before the baseline recording and at 15, 30, and 45 min after the cold pressor stress test. In control subjects, the sampling timepoints were complementary to those in subjects exposed to stress. All samples were frozen and stored at $-70^{\circ} \mathrm{C}$ until assayed. On the day of assay, the Salivette tubes were thawed at room temperature and centrifuged at $3000 \mathrm{~g}$ for $10 \mathrm{~min}$. Saliva samples were visually inspected for blood contamination and particles.

\section{Salivary cortisol}

Cortisol concentrations in saliva were measured by a chemiluminescence-based assay using an automatic analysis system (Nichols Institute Diagnostics). Briefly, a biotynilated cortisol and cortisol in the sample compete for a limited amount of chemiluminescent-labeled antibody. After an incubation period of 20 min, streptavidin-coated magnetic particles were added to the reaction mixture. During $10 \mathrm{~min}$ of incubation, streptavidincoated magnetic particles separated biotynilated cortisol onto the solid phase. Unbound labeled antibodies were then separated by aspiration of reaction mixture and washing. The wells containing the washed magnetic particles were transported to the system luminometer, which injected triggers to initiate the chemiluminescent reaction and quantitate the light. All pipeting, incubations, separations, washing steps, and measurement of light output were automatically performed by the Nichols Advantage Specialty System. Each determination was performed in duplicates; intra-assay variation was $1.5 \%$, and interassay variation was $18.6 \%$. 


\section{Statistical analyses}

Neuroendocrine changes during the procedure (i.e., changes in HR, SCR, and cortisol concentrations) were evaluated by repeated-measures ANOVA with time as a within-subject factor (four levels) and group as a between-subject factor (two levels). Due to technical problems during recording and insufficient amount of saliva collected, HR data from one participant, SCR data from six participants, and cortisol data from five participants were excluded from this analysis. Within-subject contrasts at each timepoint were used to identify when differences occurred. The number of CRs during acquisition was analyzed with a repeated-measure sANOVA with block as a within-subject factor and group as a between-subject factor. One-way ANOVAs were conducted to compare the number of spontaneous eyeblinks and amplitude of unconditioned responses across groups. Water maze performance in the control and stress groups was compared by an independent t-test (total number of failures) and repeatedmeasures ANOVAs with block as the within-subject factor and group as a between-subject factor (latency, path length, and heading error). SPSS 14.0 for Windows (SPSS Inc.) was used for all factorial analyses.

The relationships between stress response and learning performance for the stress-only group were evaluated using linear regression models, with learning performance (number of adaptive eyeblinks) as the criterion variable. Neuroendocrine responses to the cold pressor procedure (HR, SCR, cortisol) were entered as predictor variables. Statistical significance was determined using 0.05-level two-sided test. SAS 9.1 REG procedure (SAS Institute Inc.) was used for the analyses. Due to technical problems during recording and insufficient amount of saliva collected, SCR and cortisol data from two participants were excluded from this analysis.

\section{Acknowledgment}

This study was supported by the intramural research program of the National Institute of Mental Health.

\section{References}

Abercrombie, H.C., Kalin, N.H., and Davidson, R.J. 2005. Acute cortisol elevations cause heightened arousal ratings of objectively nonarousing stimuli. Emotion 5: 354-359.

Abercrombie, H.C., Speck, N.S., and Monticelli, R.M. 2006. Endogenous cortisol elevations are related to memory facilitation only in individuals who are emotionally aroused. Psychoneuroendocrinology 31: 187-196.

Akirav, I., Kozenicky, M., Tal, D., Sandi, C., Venero, C., and Richter-Levin, G. 2004. A facilitative role for corticosterone in the acquisition of a spatial task under moderate stress. Learn. Mem. 11: $188-195$.

Andreano, J.M. and Cahill, L. 2006. Glucocorticoid release and memory consolidation in men and women. Psychol. Sci. 17: 466-470.

Baker, K.B. and Kim, J.J. 2002. Effects of stress and hippocampal NMDA receptor antagonism on recognition memory in rats. Learn. Mem. 9: 58-65.

Bangasser, D.A., Santollo, J., and Shors, T.J. 2005. The bed nucleus of the stria terminalis is critically involved in enhancing associative learning after stressful experience. Behav. Neurosci. 119: 1459-1466.

Bemelmans, K.J., Goekoop, J.G., de Rijk, R., and van Kempen, G.M. 2003. Recall performance, plasma cortisol, and plasma norepinephrine in normal human subjects. Biol. Psychol. 62: 1-15.

Beylin, A.V., Gandhi, C.C., Wood, G.E., Talk, A.C., Matzel, L.D., and Shors, T.J. 2001. The role of the hippocampus in trace conditioning: Temporal discontinuity or task difficulty? Neurobiol. Learn. Mem. 76: $447-461$.

Cahill, L., Gorski, L., and Le, K. 2003. Enhanced human memory consolidation with post-learning stress: Interaction with the degree of arousal at encoding. Learn. Mem. 10: 270-274.

Christian, K.M. and Thompson, R.F. 2003. Neural substrates of eyeblink conditioning: acquisition and retention. Learn. Mem. 10: 427-455.

Clark, R.E., Manns, J.R., and Squire, L.R. 2001. Trace and delay eyeblink conditioning: Contrasting phenomena of declarative and nondeclarative memory. Psychol. Sci. 12: 304-308.

Croiset, G., Nijsen, M.J., and Kamphuis, P.J. 2000. Role of corticotropin-releasing factor, vasopressin, and the autonomic nervous system in learning and memory. Eur. J. Pharmacol.
405: $225-234$.

D’Hooge, R. and De Deyn, P.P. 2001. Applications of the Morris water maze in the study of learning and memory. Brain Res. Brain Res. Rev. 36: $60-90$

de Quervain, D.J., Roozendaal, B., and McGaugh, J.L. 1998. Stress and glucocorticoids impair retrieval of long-term spatial memory. Nature 394: 787-790.

Diamond, D.M., Fleshner, M., Ingersoll, N., and Rose, G.M. 1996. Psychological stress impairs spatial working memory: Relevance to electrophysiological studies of hippocampal function. Behav. Neurosci. 110: 661-672.

Domes, G., Heinrichs, M., Rimmele, U., Reichwald, U., and Hautzinger, M. 2004. Acute stress impairs recognition for positive words-Association with stress-induced cortisol secretion. Stress 7: 173-181.

Driscoll, I., Hamilton, D.A., Yeo, R.A., Brooks, W.M., and Sutherland, R.J. 2005. Virtual navigation in humans: The impact of age, sex, and hormones on place learning. Horm. Behav. 47: 326-335.

Duncko, R., Makatsori, A., Fickova, E., Selko, D., and Jezova, D. 2006. Altered coordination of the neuroendocrine response during psychosocial stress in subjects with high trait anxiety. Prog. Neuropsychopharmacol. Biol. Psychiatry 30: 1058-1066.

First, M.B., Spitzer, R.I., Williams, J.B.W., and Gibbon, M. 1995. Structured clinical interview for DSM-IV (SCID). American Psychiatric Association, Washington, DC.

Grillon, C. and Hill, J. 2003. Emotional arousal does not affect delay eyeblink conditioning. Brain Res. Cogn. Brain Res. 17: 400-405.

Habib, K.E., Gold, P.W., and Chrousos, G.P. 2001. Neuroendocrinology of stress. Endocrinol. Metab. Clin. North Am. 30: 695-728.

Hamilton, D.A., Driscoll, I., and Sutherland, R.J. 2002. Human place learning in a virtual Morris water task: Some important constraints on the flexibility of place navigation. Behav. Brain Res. 129: $159-170$.

Hassert, D.L., Miyashita, T., and Williams, C.L. 2004. The effects of peripheral vagal nerve stimulation at a memory-modulating intensity on norepinephrine output in the basolateral amygdala. Behav. Neurosci. 118: 79-88.

Herman, J.P., Patel, P.D., Akil, H., and Watson, S.J. 1989. Localization and regulation of glucocorticoid and mineralocorticoid receptor messenger RNAs in the hippocampal formation of the rat. Mol. Endocrinol. 3: 1886-1894.

Het, S., Ramlow, G., and Wolf, O.T. 2005. A meta-analytic review of the effects of acute cortisol administration on human memory. Psychoneuroendocrinology 30: 771-784.

Jackson, E.D., Payne, J.D., Nadel, L., and Jacobs, W.J. 2006. Stress differentially modulates fear conditioning in healthy men and women. Biol. Psychiatry 59: 516-522.

Jelici, M., Geraerts, E., Merckelbach, H., and Guerrieri, R. 2004. Acute stress enhances memory for emotional words, but impairs memory for neutral words. Int. J. Neurosci. 114: 1343-1351.

Joels, M., Pu, Z., Wiegert, O., Oitzl, M.S., and Krugers, H.J. 2006. Learning under stress: How does it work? Trends Cogn. Sci. 10: $152-158$.

Kirsch, P., Achenbach, C., Kirsch, M., Heinzmann, M., Schienle, A., and Vaitl, D. 2003. Cerebellar and hippocampal activation during eyeblink conditioning depends on the experimental paradigm: A MEG study. Neural Plast. 10: 291-301.

Kirschbaum, C., Wolf, O.T., May, M., Wippich, W., and Hellhammer, D.H. 1996. Stress- and treatment-induced elevations of cortisol levels associated with impaired declarative memory in healthy adults. Life Sci. 58: $1475-1483$.

Kopin, I.J. 1995. Definitions of stress and sympathetic neuronal responses. Ann. N. Y. Acad. Sci. 771: 19-30.

Luine, V., Villegas, M., Martinez, C., and McEwen, B.S. 1994. Repeated stress causes reversible impairments of spatial memory performance. Brain Res. 639: 167-170.

Lupien, S.J., Gaudreau, S., Tchiteya, B.M., Maheu, F., Sharma, S., Nair, N.P., Hauger, R.L., McEwen, B.S., and Meaney, M.J. 1997. Stress-induced declarative memory impairment in healthy elderly subjects: Relationship to cortisol reactivity. J. Clin. Endocrinol. Metab. 82: 2070-2075.

Lupien, S.J., Gillin, C.J., and Hauger, R.L. 1999. Working memory is more sensitive than declarative memory to the acute effects of corticosteroids: A dose-response study in humans. Behav. Neurosci. 113: $420-430$.

McGaugh, J.L. 2000. Memory-A century of consolidation. Science 287: 248-251

McRae, A.L., Saladin, M.E., Brady, K.T., Upadhyaya, H., Back, S.E., and Timmerman, M.A. 2006. Stress reactivity: Biological and subjective responses to the cold pressor and trier social stressors. Hum. Psychopharmacol. 21: 377-385.

Morimoto, M., Morita, N., Ozawa, H., Yokoyama, K., and Kawata, M.

\section{Learning \& Memory}


1996. Distribution of glucocorticoid receptor immunoreactivity and mRNA in the rat brain: An immunohistochemical and in situ hybridization study. Neurosci. Res. 26: 235-269.

Morris, R.G., Garrud, P., Rawlins, J.N., and O'Keefe, J. 1982. Place navigation impaired in rats with hippocampal lesions. Nature 297: 681-683.

Newcomer, J.W., Selke, G., Melson, A.K., Hershey, T., Craft, S., Richards, K., and Alderson, A.L. 1999. Decreased memory performance in healthy humans induced by stress-level cortisol treatment. Arch. Gen. Psychiatry 56: 527-533.

Oitzl, M.S. and de Kloet, E.R. 1992. Selective corticosteroid antagonists modulate specific aspects of spatial orientation learning. Behav. Neurosci. 106: 62-71.

Okuda, S., Roozendaal, B., and McGaugh, J.L. 2004. Glucocorticoid effects on object recognition memory require training-associated emotional arousal. Proc. Natl. Acad. Sci. 101: 853-858.

Pacak, K. and Palkovits, M. 2001. Stressor specificity of central neuroendocrine responses: Implications for stress-related disorders. Endocr. Rev. 22: 502-548.

Park, C.R., Campbell, A.M., and Diamond, D.M. 2001. Chronic psychosocial stress impairs learning and memory and increases sensitivity to yohimbine in adult rats. Biol. Psychiatry 50: 994-1004

Putman, P., Van Honk, J., Kessels, R.P., Mulder, M., and Koppeschaar, H.P. 2004. Salivary cortisol and short and long-term memory for emotional faces in healthy young women. Psychoneuroendocrinology 29: 953-960.

Roozendaal, B. 2002. Stress and memory: Opposing effects of glucocorticoids on memory consolidation and memory retrieval. Neurobiol. Learn. Mem. 78: 578-595.

Roozendaal, B., Carmi, O., and McGaugh, J.L. 1996. Adrenocortical suppression blocks the memory-enhancing effects of amphetamine and epinephrine. Proc. Natl. Acad. Sci. 93: 1429-1433.

Roozendaal, B., Hahn, E.L., Nathan, S.V., de Quervain, D.J., and McGaugh, J.L. 2004a. Glucocorticoid effects on memory retrieval require concurrent noradrenergic activity in the hippocampus and basolateral amygdala. J. Neurosci. 24: 8161-8169.

Roozendaal, B., McReynolds, J.R., and McGaugh, J.L. 2004b. The basolateral amygdala interacts with the medial prefrontal cortex in regulating glucocorticoid effects on working memory impairment. $J$. Neurosci. 24: 1385-1392.

Roozendaal, B., Okuda, S., Van der Zee, E.A., and McGaugh, J.L. 2006. Glucocorticoid enhancement of memory requires arousal-induced noradrenergic activation in the basolateral amygdala. Proc. Natl. Acad. Sci. 103: 6741-6746.

Sandi, C., Loscertales, M., and Guaza, C. 1997. Experience-dependent facilitating effect of corticosterone on spatial memory formation in the water maze. Eur. J. Neurosci. 9: 637-642.

Selye, H. 1975. The stress of life, revised ed. McGraw Hill Book, New York.

Servatius, R.J. and Beck, K.D. 2003. Facilitated acquisition of the classically conditioned eyeblink response in male rats after systemic IL-1ß. Integr. Physiol. Behav. Sci. 38: 169-178.

Servatius, R.J., Beck, K.D., Moldow, R.L., Salameh, G., Tumminello, T.P., and Short, K.R. 2005. A stress-induced anxious state in male rats: Corticotropin-releasing hormone induces persistent changes in associative learning and startle reactivity. Biol. Psychiatry 57: $865-872$.

Shors, T.J. 2001. Acute stress rapidly and persistently enhances memory formation in the male rat. Neurobiol. Learn. Mem. 75: 10-29.

Shors, T.J. and Mathew, P.R. 1998. NMDA receptor antagonism in the lateral/basolateral but not central nucleus of the amygdala prevents the induction of facilitated learning in response to stress. Learn. Mem. 5: 220-230.

Shors, T.J. and Matzel, L.D. 1997. Long-term potentiation: What's learning got to do with it? Behav. Brain Sci. 20: 597-614. discussion 614-655

Shors, T.J., Chua, C., and Falduto, J. 2001. Sex differences and opposite effects of stress on dendritic spine density in the male versus female hippocampus. J. Neurosci. 21: 6292-6297.

Steidl, S., Mohi-uddin, S., and Anderson, A.K. 2006. Effects of emotional arousal on multiple memory systems: Evidence from declarative and procedural learning. Learn. Mem. 13: 650-658.

Takahashi, T. 2005. Social memory, social stress, and economic behaviors. Brain Res. Bull. 67: 398-402.

Tapp, W., Servatius, R., Hunt, J., and Powell, D.A. 1997. Vagal activity predicts eyeblink conditioning in human subjects. Neuroreport 8: $1203-1207$.

Tracy, J.A., McFall, R.M., and Steinmetz, J.E. 2005. Effects of emotional valence and arousal manipulation on eyeblink classical conditioning and autonomic measures. Integr. Physiol. Behav. Sci. 40: 45-54.

van Eekelen, A.P., Kerkhof, G.A., and van Amsterdam, J.G. 2003. Circadian variation in cortisol reactivity to an acute stressor. Chronobiol. Int. 20: $863-878$.

Vining, R.F., McGinley, R.A., and Symons, R.G. 1983. Hormones in saliva: Mode of entry and consequent implications for clinical interpretation. Clin. Chem. 29: 1752-1756.

Weiss, C., Sametsky, E., Sasse, A., Spiess, J., and Disterhoft, J.F. 2005. Acute stress facilitates trace eyeblink conditioning in C57BL/6 male mice and increases the excitability of their CA1 pyramidal neurons. Learn. Mem. 12: 138-143.

Wolf, O.T. 2003. HPA axis and memory. Best. Pract. Res. Clin. Endocrinol. Metab. 17: 287-299.

Woodson, J.C., Macintosh, D., Fleshner, M., and Diamond, D.M. 2003. Emotion-induced amnesia in rats: Working memory-specific impairment, corticosterone-memory correlation, and fear versus arousal effects on memory. Learn. Mem. 10: 326-336.

Wright, R.L. and Conrad, C.D. 2005. Chronic stress leaves novelty-seeking behavior intact while impairing spatial recognition memory in the Y-maze. Stress 8: 151-154.

Yang, Y., Cao, J., Xiong, W., Zhang, J., Zhou, Q., Wei, H., Liang, C., Deng, J., Li, T., Yang, S., et al. 2003. Both stress experience and age determine the impairment or enhancement effect of stress on spatial memory retrieval. J. Endocrinol. 178: 45-54.

Zorawski, M., Cook, C.A., Kuhn, C.M., and LaBar, K.S. 2005. Sex, stress, and fear: Individual differences in conditioned learning. Cogn. Affect. Behav. Neurosci. 5: 191-201.

Received November 21, 2006; accepted in revised form March 13, 2007. 


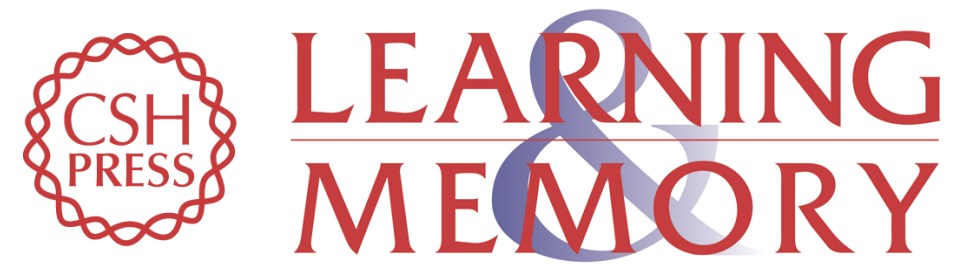

\section{Acute exposure to stress improves performance in trace eyeblink conditioning and spatial learning tasks in healthy men}

Roman Duncko, Brian Cornwell, Lihong Cui, et al.

Learn. Mem. 2007, 14:

Access the most recent version at doi:10.1101//m.483807

References This article cites 67 articles, 17 of which can be accessed free at: http://learnmem.cshlp.org/content/14/5/329.full.html\#ref-list-1

License

Email Alerting Receive free email alerts when new articles cite this article - sign up in the box at the Service top right corner of the article or click here. 